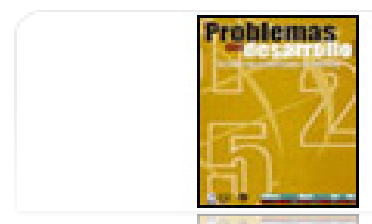

Problemas del Desarrollo. Revista Latinoamericana de Economía

ISSN: 0301-7036

revprode@servidor.unam.mx

Universidad Nacional Autónoma de México

México

Morales Barragán, Federico

Territorio, redes e instituciones: una experiencia en regiones marginadas de Chiapas

Problemas del Desarrollo. Revista Latinoamericana de Economía, vol. 35, núm. 137, 2004, pp. 59-76

Universidad Nacional Autónoma de México

Distrito Federal, México

Disponible en: http://www.redalyc.org/articulo.oa?id=11825947004

Cómo citar el artículo

Número completo

- Más información del artículo

Página de la revista en redalyc.org

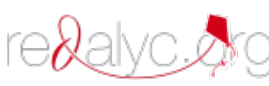

Sistema de Información Científica

Red de Revistas Científicas de América Latina, el Caribe, España y Portugal

Proyecto académico sin fines de lucro, desarrollado bajo la iniciativa de acceso abierto 


\title{
TERRITORIO, REDES E INSTITUCIONES: UNA EXPERIENCIA EN REGIONES MARGINADAS DE CHIAPAS
}

\author{
Federico Morales Barragán*
}

Fecha de recepción: 30 de octubre de 2003. Fecha de aceptación: 22 de junio de 2004.

\begin{abstract}
Resumen
El propósito de este trabajo es mostrar que los planteamientos sobre la innovación ofrecen orientaciones pertinentes para analizar una experiencia de tipo productivo en la sierra de Chiapas. Para ello se construye, en primer lugar, un marco de referencia de este debate mediante el enlace de las nociones de territorio, conversión del conocimiento y articulación globallocal. Luego se ofrece una discusión sobre los mecanismos de cooperación que favorecen el desarrollo de las innovaciones. Finalmente se reconstruye la historia de la experiencia citada anteriormente. Dicha reconstrucción permite apreciar el vínculo entre las innovaciones productivas y los mecanismos de cooperación que contribuyen a su desarrollo.
\end{abstract}

Palabras clave: medio innovador, sistemas de innovación, conocimiento tácito, conocimiento codificado, dinámica institucional.

\begin{abstract}
This study aims to show that the approaches to innovation offer orientations pertinent for analyzing an experience of the productive type in the Chiapas sierra. For this purpose, in the first place, a reference framework was constructed for this debate using the concepts of territory, knowledge conversion and global-local articulation. Following that, a discussion is presented on the mechanisms of cooperation favoring the development of innovations. Finally, a history of the previously cited experience is reconstructed. This reconstruction allows us to appreciate the link between productive innovations and the mechanisms of cooperation contributing to their development.
\end{abstract}

Key terms: innovative medium, innovative systems, tacit knowledge, codified knowledge, institutional dynamics.

* Investigador del Programa de Investigaciones Multidisciplinarias sobre Mesoamérica y el Sureste, Instituto de Investigaciones Antropológicas (PROIMMSE-IIA-UNAM). Correo electrónico: fmorales@servidor.unam.mx 


\section{Résumé}

Le but de ce travail est de montrer que les travaux préliminaires sur l'innovation offrent des orientations pertinentes pour l'analyse d'une expérience de type productif dans la sierra du Chiapas. Pour ce faire, on construit dans un premier temps, un cadre de référence de ce débat moyennant un lien entre les notions de territoire, de conversion, des connaissances, et d'articulation globale locale. On offre ensuite une discussion sur les mécanismes de coopération qui favorisent le développement des innovations. On reconstruit finalement l'histoire de l'expérience mentionnée antérieurement. Cette reconstruction permet d'apprécier le lien entre les innovations productives et les mécanismes de coopération qui contribuent à leur développement.

Mots clés: moyen, innovateur, systèmes d'innovation, connaissance tacite, connaissance codifiée, dynamique institutionnelle.

\section{Resumo}

O propósito deste trabalho é mostrar que os planejamentos que priorizam a inovação oferecem orientações pertinentes para análise de uma experiência de tipo produtiva na serra de Chiapas. Para isso, em primeiro lugar, é estabelecido um marco de referência para o debate, mediante $o$ enlace das noções de território, conversão do conhecimento e articulação global-local. A seguir, se discute os mecanismos de cooperação que favorecem o desenvolvimento das inovações. E por fim, se reconstrói a história da experiência citada anteriormente. Essa reconstrução permite apreciar o vínculo entre as inovações produtivas e os mecanismos de cooperação que contribuem para o desenvolvimento.

Palavras-chave: meio inovador, sistemas de inovação, conhecimento tácito, conhecimento codificado, dinâmica institucional. 


\section{Introducción}

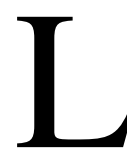

a innovación constituye uno de los factores clave de los procesos de desarrollo económico: "La generación de la novedad y la absorción de ésta dentro de la economía y las estructuras sociales es quizás la cuestión dinámica central a la que nos debemos dirigir si el cambio ha de ser explicado" (Metcalfe, 1995:1561). Por lo anterior, no es casual que el origen y difusión de las innovaciones, así como su impacto en el desempeño económico, sea uno de los temas relevantes en las discusiones sobre reestructuración económica y desarrollo.

En la última década han proliferado estudios que, con diversos enfoques, destacan el carácter interactivo de los procesos de innovación (Cooke y Morgan, 2000; Edquist, 1997; Fischer y Frölich, 2001; Malecki y Oinas, 1998; Metcalf, 1995; Moulaert y Sekia, 1999 y Vázquez, 1999). La creación y difusión de las innovaciones — antes concebidas como dos fases disociadas en que los actores involucrados tenían una interacción limitada — se analizan ahora como parte integral de un proceso único que genera redes de intercambio y cooperación entre actores diversos.

Hasta ahora el estudio de estos fenómenos se ha dirigido a experiencias que tienen lugar en países desarrollados. A pesar de que el tema de las innovaciones domina el debate sobre reestructuración económica y desarrollo, las regiones subdesarrolladas no han recibido suficiente atención en lo que respecta al análisis de las nuevas formas de producir y difundir las innovaciones. Inclusive, se considera improbable que ocurran en regiones marginadas debido a los rasgos generales de éstas: infraestructura precaria, población con bajos niveles de calificación y escasa diversificación de sus actividades económicas, en general, con bajos niveles de calidad y productividad.

El propósito de este trabajo es mostrar que el debate contemporáneo sobre la innovación pone de relieve el carácter interactivo de los procesos de innovación y que este rasgo es fundamental en el desarrollo de una experiencia de innovación productiva de una región marginada del estado de Chiapas. Para ilustrar este argumento primero se construye el marco de referencia de este debate mediante la articulación de las nociones de territorio, conversión del conocimiento y articulación global-local. Luego se estudian los mecanismos de cooperación que favorecen el desarrollo de las innovaciones. Finalmente, se analizan etapas de una experiencia de innovación productiva en la región sierra del estado de Chiapas, que permite apreciar el vínculo entre las innovaciones productivas y los mecanismos de cooperación que contribuyen a su desarrollo. 


\section{Territorio, conversión del conocimiento y articulación global-local}

Los años setenta marcan el inicio de un proceso de reestructuración económica diferenciado regionalmente (Cossentino et al., 1996; Oman, 1994; Vázquez, 1993 y Vence, 1995). Estos cambios se han expresado, entre otros aspectos, en el desarrollo de sistemas de organización productiva más flexibles que se adaptan con mayor facilidad a entornos que cambian rápidamente. Los sistemas jerárquicos han cedido paulatinamente el paso a otros organizados horizontalmente en los que las redes de cooperación juegan un papel cada vez más importante (Fischer, 1999; Maillat y Kebir, 1998 y Vázquez, 1999). Cooperar para competir se ha convertido en la divisa de las nuevas formas de organización productiva.

El surgimiento de estas redes de cooperación revela, asimismo, cambios en la concepción del territorio, que actualmente no se consideran depósitos de recursos genéricos susceptibles de utilizarse, sino ámbitos de construcción de recursos específicos. De ahí que las decisiones territoriales de las unidades económicas no se reduzcan sólo a la identificación de recursos disponibles, sino a un proceso de construcción de los mismos que no se restringe al ámbito específico de dichas unidades, sino que involucra la interacción de éstas con su entorno (Veltz, 1993).

[Un] territorio adquiere carácter competitivo si puede afrontar la competencia del mercado y garantizar al mismo tiempo la viabilidad medioambiental, económica, social y cultural, aplicando lógicas de red y de articulación inter-territorial. En otros términos, la competitividad territorial supone: la toma en cuenta de los recursos del territorio en la búsqueda de la coherencia global; la implicación de los agentes e instituciones; la integración de los sectores de actividad en una lógica de innovación; la cooperación de los otros territorios y la articulación con las políticas regionales, nacionales y [continentales] con el contexto global (Observatorio Europeo LEADER, 2001:5).

Becattini y Rullani (1996) señalan que la producción de bienes y servicios no puede separarse de la reproducción del sistema social en conjunto. Además de los bienes y servicios se coproducen valores, conocimiento, instituciones y ambiente físico necesarios para darle continuidad. Por ello, la viabilidad del sistema involucra aspectos económicos, sociales, culturales, institucionales y ambientales.

Por lo anterior, el análisis sectorial se complementa con el territorial, en la medida en que éste último ofrece una perspectiva multidimensional de los procesos de cambio. Este enfoque contrasta con el expresado por el análisis económico convencional que concibe al territorio como un espacio abstracto descrito mediante funciones de costo de transporte o, en general, mediante funciones de accesibilidad (Beckmann y Thisse, 1986; Takayama y Labys, 1986).

El estudio de la dinámica territorial, es decir, aquella mediante la cual se construyen los recursos específicos de cada territorio, ofrece una perspectiva más rica para analizar 
los procesos de innovación ya que contempla las interacciones en los diversos ámbitos donde se configuran.

Nonaka y Takeuchi (1999) proponen un modelo de construcción del conocimiento para analizar la dinámica de la innovación que es coherente con esta perspectiva. Su modelo establece que el conocimiento humano se crea y expande mediante la interacción social de los conocimientos tácitos y explícito. El primero es personal y específico de un contexto. Alude a prácticas comunes, modos de interpretación, percepciones y sistemas de valores, por lo cual es difícil de formalizar y, en ocasiones, de comunicar. El conocimiento explícito o codificado, en cambio, se transmite mediante un lenguaje formal y sistemático.

Para estos autores existen cuatro formas de conversión del conocimiento que involucran a los mencionados: de tácito a tácito (socialización); de tácito a explícito (exteriorización); de explícito a explícito (combinación) y, finalmente, de explícito a tácito (interiorización).

La socialización es un proceso que consiste en compartir experiencias y, por tanto, crear conocimiento tácito tal como los modelos mentales compartidos y las habilidades técnicas. Un individuo puede adquirir conocimiento tácito directamente de otros sin usar el lenguaje sino a través de la observación, la imitación y la práctica [...]

La exteriorización es un proceso a través del cual se enuncia el conocimiento tácito en forma de conceptos explícitos [bajo la forma] de metáforas, analogías, conceptos, hipótesis o modelos [...]

La combinación es un proceso de sistematización de conceptos con el que se genera un sistema de conocimiento [...] La reconfiguración de la información existente que se lleva a cabo clasificando, añadiendo, combinando y categorizando el conocimiento explícito (como en bases de datos de computadora), puede conducir a un nuevo conocimiento [...]

La interiorización [...] está muy relacionada con el 'aprender haciendo'. Cuando las experiencias son internalizadas en la base de conocimiento tácito de los individuos a través de la socialización, la exteriorización y la combinación, en la forma de modelos mentales compartidos y know-how técnico, se vuelven activos muy valiosos (Nonaka y Takeuchi, 1999:70, 72, 76 y 78, énfasis en el original).

El modelo de Nonaka y Takeuchi es compatible también con el enfoque general propuesto por Rullani (1997) sobre la articulación global-local. El tratamiento de Rullani brinda una perspectiva útil para estudiar los procesos de innovación poniendo de relieve el ámbito territorial, que no está suficientemente clara en el modelo anterior.

En general, la dimensión global se refiere a los procesos de producción, transferencia y uso del conocimiento que carecen de un referente territorial explícito y se trasladan de un lugar a otro con el propósito de replicarse. Por su parte, la dimensión local alude a la forma en que estos procesos encarnan en un territorio determinado. De ahí que la articulación global-local, más que la simple relación, puede concebirse como un flujo entre procesos de recontextualización (de lo global hacia lo local) y descontextualización (de lo 
local hacia lo global) de la producción, transferencia y uso del conocimiento (Rullani, 1997).

Se aprecia que el modelo de Nonaka y Takeuchi complementa el enfoque de Rullani a propósito de la articulación global-local. Las formas de conversión a las que hacen referencia los primeros se vinculan con los procesos de descontextualización y recontextualización de la producción, transferencia y uso del conocimiento. El conocimiento tácito vinculado a las prácticas y saberes de un territorio se descontextualiza y convierte en conocimiento codificado mediante la exteriorización. A su vez, mediante la interiorización (recontextualización), un grupo de productores puede adecuar procedimientos y técnicas no generadas en su territorio.

Las consideraciones anteriores ofrecen un marco de referencia para el estudio de los procesos de innovación, en el que destacan las modalidades de intercambio, asimilación y difusión del conocimiento. Esta situación exige reflexionar sobre los mecanismos de cooperación que favorecen el desarrollo de las innovaciones.

\section{Cooperación e innovaciones: algunos elementos del debate contemporáneo}

Moulaert y Sekia (1999) proponen el término genérico modelos territoriales de innovación para nombrar aquellos en los que la dinámica de las instituciones locales juega un papel destacado. Bajo esta noción se engloban las contribuciones del Grupo de Investigación Europeo sobre el Medio Innovador (GREMI), las cuales destacan el potencial institucional endógeno de un territorio para generar una dinámica innovadora en las empresas. También se encuentran los aportes del modelo de distritos industriales, que señala la importancia de la cooperación y la asociación en los procesos innovadores. Otro enfoque se ubica en la tradición de los sistemas de innovación, sea bajo el traslado de la coordinación institucional de los ámbitos nacional o sectorial al regional, o de la interpretación evolucionista, a escala regional, de la economía del aprendizaje. Otro más es el de los nuevos espacios industriales, que parte de la tradición de la Escuela Californiana de Geografía Económica y, por último, están las contribuciones sobre conglomerados espaciales de innovación que se vinculan con las discusiones de Porter sobre los conglomerados de innovación (Moulaert y Sekia, 1999).

En esta sección se hace referencia a contribuciones de ambos enfoques: medio innovador y sistemas de innovación. En ellos se aprecian perspectivas y temas afines que ponen de relieve el carácter interactivo de los procesos contemporáneos de innovación.

Antes de dar paso a esta discusión conviene considerar la definición propuesta por Tijssen que, según Fischer, abarca los rasgos más importantes de las estructuras cooperativas denominadas genéricamente redes de innovación. 
[Una red es] un sistema evolutivo de dependencia mutua basado en relaciones de recursos [; su] carácter sistémico es el resultado de interacciones, procesos, procedimientos e institucionalización. Las actividades dentro de tal tipo de red involucran la creación, combinación, intercambio, transformación, absorción y explotación de recursos dentro de un amplio rango de relaciones formales e informales (Fischer, 1999:17-18).

\section{Medio innovador}

Maillat y Kebir (1998) señalan que los procesos de aprendizaje son la base para construir los recursos inmateriales que involucra la innovación y para mantener las ventajas competitivas de un territorio. Desde esta perspectiva, la expansión y difusión del conocimiento no sólo descansa en los sistemas convencionales de investigación (universidades, centros y programas de investigación y desarrollo); en este proceso tiene especial relevancia la constitución de un ambiente institucional que propicie la cooperación, esto es, el intercambio permanente de información entre los diversos actores a los que convoca la solución de un problema común.

El ambiente institucional al que hacen referencia remite a la noción de medio innovador (MI), ambiente que se caracteriza por la presencia de un conjunto de saberes, normas, reglas y valores que guían el comportamiento de los actores y sus relaciones. Estos elementos se configuran a partir de la interacción entre unidades productivas, centros de investigación y formación, instituciones financieras, asociaciones socio-profesionales e instituciones de gobierno (Maillat, 1996).

De acuerdo con Maillat y Grosjean (1999), el MI comprende los siguientes aspectos: a) entidad territorial con cierto grado de unidad que se refleja en rasgos de comportamiento identificables; $b$ ) grupo de actores (empresas, instituciones de investigación, adiestramiento y educación, autoridades locales e individuos calificados) que necesitan cierta independencia para tomar sus decisiones; $c$ ) aspectos materiales (empresas e infraestructura), inmateriales (know-how, reglas) e institucionales específicos; $d$ ) lógica organizacional (capacidad de cooperar) dirigida a un mejor uso de los recursos creados en común y e) lógica de aprendizaje que caracteriza la capacidad de los actores de modificar su comportamiento en correspondencia con los cambios tecnológicos y de mercado.

El MI se refiere, entonces, al acervo social y cultural específico y que se vincula con el desarrollo de los sistemas productivos territoriales. Este último concepto sintetiza el objeto de análisis de un buen número de las reflexiones contemporáneas sobre la economía regional, y se utiliza

para indicar la multiplicidad de formas de organización espacial de la producción en las que juegan un papel positivo y significativo los efectos de sinergia y de proximidad: desde los sistemas locales de pequeña empresa hasta los distritos tecnológicos, desde las áreas sistema hasta los 'milieux innovateurs'(Bramanti y Maggioni, 1997:23); 
Vinculada a la noción de MI se encuentra la de redes de innovación. Diversas contribuciones asociadas al GREMI insisten en la complementariedad de los conceptos de redes locales y translocales para entender la amplitud del carácter interactivo de los procesos de innovación (Bramanti y Ratti, 1997). Las redes de innovación

se definen como un conjunto coordinado de actores heterogéneos pero profesionales (laboratorios públicos, centros de investigación técnica, empresas, etc.) que participan colectivamente en la concepción, la elaboración, la producción y la difusión de procedimientos de producción, de bienes y de servicios, que dan lugar a una transacción mercantil (Maillat, 1996:13).

Estas redes presentan vínculos directos y relaciones no jerárquicas entre los actores, y su tendencia a coordinarse obedece a la posibilidad de reducir riesgos y costos asociados a los procesos de innovación, puesto que ninguno de ellos posee en lo individual los recursos necesarios para ponerlos en marcha. Esta opinión es similar a la expresada por Fischer: "El incremento de la complejidad, los riesgos y los costos en la innovación destacan el papel de la colaboración y el establecimiento de redes en el proceso de innovación para reducir el riesgo moral y los costos de transacción" (Fischer, 2001:209).

Merece recordarse que, como señalan Bramanti y Ratti (1997), diversas contribuciones vinculadas con el GREMI han insistido en que las redes de innovación no se restringen al ámbito local.

Las redes de innovación asociadas a un MI no parten, necesariamente, de un problema claramente definido. La innovación asume un carácter multifuncional en el que no se pueden evaluar a priori los costos y beneficios de cada uno de los participantes: "Estas redes multifuncionales proceden por tanteo, ensayo y error y reorientaciones sucesivas del proyecto" (Maillat, 1996:14). Para Maillat estas redes constituyen una fuente de acumulación y de actualización de las potencialidades creativas del MI.

\section{Sistemas de innovación}

De acuerdo con Fischer (2001), el enfoque de los sistemas de innovación ofrece un marco conceptual que sostiene que la innovación no sólo se gesta en las actividades de investigación y desarrollo: "Un sistema de innovación puede ser pensado como un conjunto de actores tales como empresas, otras organizaciones e instituciones que interactúan en la generación, difusión y uso de un conocimiento nuevo - y económicamente útil— en el proceso de producción" (Fischer, 2001:207).

Estos sistemas involucran una amplia gama de relaciones que comprenden desde estructuras formales basadas en regulaciones y contratos hasta contactos personales en los cuales la proximidad territorial y las relaciones de confianza desempeñan un papel sobresaliente. 
Su funcionamiento ha evidenciado la imposibilidad de separar la creación y difusión del conocimiento. En la actualidad se reconoce que estas facetas: "usualmente emergen como resultado de un proceso interactivo y colectivo dentro de una telaraña de conexiones personales e institucionales que evolucionan a lo largo del tiempo" (Fischer, 2001:204). Dichas conexiones pueden referirse a relaciones entre: a) productores y consumidores, como pueden ser los vínculos hacia delante entre los primeros y sus distribuidores, revendedores y usuarios finales; $b$ ) productores y sus proveedores de insumos o productos intermedios; $c$ ) productores y proveedores de servicios; $d$ ) grupos de productores que incluyen acuerdos de coproducción y, por último, $e$ ) productores y centros de investigación o universidades que establecen acuerdos de consultoría o de investigaciones aplicadas (Fischer, 1999 y 2001).

Las nociones de proximidad geográfica y tecnológica han sido utilizadas para explicar los requisitos para la constitución de un sistema de innovación. Echeverri-Carroll y Brennan (1999) señalan la importancia de la proximidad geográfica en la transmisión del conocimiento, a pesar de que en la actualidad se dispone de medios de comunicación que parecen restarle peso. Este argumento destaca la influencia de la aglomeración en el proceso innovador, en virtud de los beneficios que ofrece: disponibilidad de trabajo y servicios especializados, ahorros en costos de transporte y economías de escala. En correspondencia con esta situación: "las innovaciones en las firmas localizadas en ciudades con una relativamente menor acumulación de conocimiento dependen de relaciones con universidades y otras firmas de alta tecnología localizadas en otra parte" (Echeverri-Carroll y Brennan, 1999:47).

La proximidad tecnológica concierne a las interdependencias horizontales y verticales de las relaciones productivas. Maillat y Grosjean (1999) muestran que los sistemas productivos territoriales que operan con una lógica territorial (cultivo de relaciones de cooperación y competencia que generan sinergias y complementariedades) tienen mayores márgenes para conducir su propio proceso de desarrollo. Sin embargo, esta lógica coexiste muchas veces con una funcional (organización jerárquica y vertical de los procesos productivos que se localizan geográficamente en función de la reducción de sus costos de operación).

Se aprecia que el énfasis en el carácter interactivo y no lineal del proceso innovador, que postulan los enfoques del Mi y de los sistemas de innovación, otorga especial importancia al funcionamiento de las instituciones que regulan las relaciones entre los actores de una red de innovación. Esta perspectiva cobra mayor relevancia si se considera que las capacidades específicas de las organizaciones, esto es, los componentes tácitos del conocimiento (prácticas comunes, modos de interpretación, percepciones y sistemas de valores) sólo pueden compartirse mediante relaciones establecidas en el ámbito de las redes sociales (Fischer, 2001). 
[La] comprensión de la innovación como un proceso social, no lineal e interactivo ha dado énfasis al papel jugado por las estructuras socio-culturales en el desarrollo regional como prerrequisitos necesarios para que las regiones sean innovativas y competitivas en la economía global post-Fordista (Asheim y Cooke, 1998:145).

\section{Una experiencia de innovación productiva en la Sierra de Chiapas ${ }^{1}$}

La historia del Grupo K'Nan Choch es la de una experiencia de innovación productiva cuyos resultados se expresan fundamentalmente en dos dimensiones. Por una parte, en el diseño y producción de una innovación de producto, un bien agroindustrial altamente diferenciado (papas criollas orgánicas crudas, cortadas a la francesa). Por otra, en el aprendizaje colectivo de una forma específica de funcionamiento: construir vínculos de cooperación facilita el intercambio de conocimiento y propicia el surgimiento y desarrollo de este tipo de proyectos.

El origen del Grupo K'Nan Choch — que en lengua mam significa "Nuestra Madre Tierra"- - se remonta al año 1988, cuando un grupo de catequistas de la diócesis de Tapachula promueve la realización de talleres de reflexión con productores de hortalizas de la región sierra, en el estado de Chiapas. El propósito de esos talleres fue identificar alternativas a su situación económica y laboral que, caracterizada por un flujo precario de ingresos derivado de una actividad agrícola de subsistencia, los conducía a ocuparse como jornaleros en las fincas cafetaleras de la sierra o del Soconusco.

Como resultado de estos talleres, en 1988 los agricultores deciden formar un grupo de trabajo que establece como objetivos mejorar la calidad de sus tierras y obtener mayores ingresos para los socios. A partir de entonces, se inicia un largo recorrido que los llevará, primero, a constituir el Grupo K'Nan Choch, que integra a 169 agricultores distribuidos en cinco municipios de la región (Bejucal de Ocampo, La Grandeza, Mazapa de Madero, Motozintla y El Porvenir) y más adelante a concluir, en el año 2001, el diseño y las pruebas experimentales del proceso técnico para producir hortalizas orgánicas $T a k-N a_{\mathrm{MR}}$ papa criolla a la francesa. El rasgo específico que convierte a este producto en una innovación es que las papas se presentan crudas, no precocidas y congeladas.

El análisis de esta experiencia muestra el vínculo entre la generación de una innovación de producto y los mecanismos de cooperación que contribuyen a su desarrollo. Situación que destaca el carácter interactivo de este proceso de innovación.

En este apartado se analiza la experiencia del grupo destacando la influencia de las instituciones regionales en la formación y funcionamiento de los vínculos de cooperación impulsados por él. De acuerdo con Fischer (2001), se entiende por instituciones regionales tanto las normas (formales y no formales) como las organizaciones que operan en donde se desarrolla esta experiencia. También se considera la influencia de organizaciones

Esta sección se basa en los resultados de la investigación reportada en Morales (2003). 
no regionales, como el Consejo Nacional de Ciencia y Tecnología (CONACYT), debido a su vinculación con proyectos del grupo.

El análisis de la experiencia del grupo se lleva a cabo con base en una propuesta que articula dos dimensiones: la dinámica institucional y la interacción social entre los conocimientos tácito y explícito. La primera se expresa en la dinámica de: $a$ ) aspectos internos que rigen las relaciones dentro del grupo, $b$ ) las interacciones entre el grupo y el resto de las organizaciones.

Esta acepción de la dinámica institucional se relaciona con la noción de espacio de soporte que, junto con las nociones de espacio de producción y de mercado, son asumidas por los enfoques sobre el medio innovador y los sistemas locales de producción para describir el funcionamiento de las empresas, enfatizando que su evolución no puede disociarse de la evolución de su entorno (Conti y Taylor, 1997). El espacio de soporte comprende relaciones de carácter no mercantil como:

Relaciones estratégicas-organizacionales, determinadas por acuerdos entre la compañía y sus socios, las cuales son puestas en práctica en el intercambio de información, en alianzas y en integraciones parciales;

relaciones privilegiadas, entre la compañía y otros actores que operan en el espacio territorial en cuestión (instituciones públicas, privadas o asociaciones semipúblicas);

relaciones genéticas-estructurales, relaciones concernientes a los términos de organización de aquellos factores inmateriales de producción (fuentes de capital, acceso a redes de know-how tecnológico, cultura empresarial y capital humano) que constituyen el milieu [...] (Conti y Taylor, 1997: 33, énfasis en el original).

La segunda dimensión, presentada en el primer apartado, se refiere al modelo propuesto por Nonaka y Takeuchi (1999) sobre el intercambio del conocimiento y el desarrollo de las innovaciones.

Esta propuesta, que integra la dinámica institucional y la interacción social entre los conocimientos tácito y explícito, permite identificar, por una parte, los ámbitos de intercambio de conocimiento donde se desarrolló la innovación de producto del grupo y por la otra, valorar cómo influye la dinámica institucional en la formación y desarrollo de dichos ámbitos de interacción.

Los sucesos analizados se ubican en dos etapas centrales en la experiencia de innovación productiva del grupo: conversión hacia las actividades agroindustriales, y experimentación y rediseño. La primera se caracteriza por el desplazamiento del eje de sus actividades: de la producción de papas orgánicas a su procesamiento agroindustrial; la segunda, se caracteriza por la experimentación orientada a producir satisfactoriamente su primer bien agroindustrial. A continuación se analizan estas dos etapas. 


\section{Conversión hacia las actividades agroindustriales}

Esta etapa se descompone en dos grandes sucesos. El primero de ellos puede considerarse el detonador de la conversión, mientras que el segundo se refiere al establecimiento de bases para desempeñarse como productores de bienes agroindustriales. Este segundo suceso se divide, a su vez, en tres que se desarrollan paralelamente: diseño del producto, establecimiento de instalaciones y adquisición de equipos, y cumplimiento con la normatividad para producir y comercializar estos bienes. Por razones de espacio sólo se analiza el diseño del producto.

Con respecto al detonador de la conversión, es el momento en que los asesores y directivos del grupo reciben información, por conducto del representante de la empresa Superior Fud, sobre la demanda de hortalizas precocidas y congeladas. Esta información determina el rumbo futuro de las iniciativas de la organización.

Este encuentro y las decisiones que toman a partir del mismo son resultado de la confluencia de los siguientes factores. Primero, el grupo dispone de personal cuya función es buscar opciones de comercialización, lo que les da contacto permanente con las tendencias del mercado. Debe tomarse en cuenta que la experiencia laboral de la persona encargada de estas actividades y la forma responsable en que ejerce sus funciones se convierten en activos muy valiosos para la organización. Ambos aspectos se materializan en iniciativas que contribuyen continuamente a ampliar la perspectiva del grupo e impulsar su desarrollo.

En segundo lugar, el grupo ha establecido las bases formales para evaluar sus actividades y buscar alternativas a los problemas que enfrentan.

La organización tiene una asamblea general que se celebra cada 24 de marzo. También está la directiva que cambia cada dos años y que se organiza bajo comités. Los miembros del comité técnico continúan, son 7 personas que ya se han capacitado. Puede haber reelección por dos años. [Además está] la junta de delegados, que reúne mensualmente a los representantes por zona y a los comités. Esta junta ahora se llama consejo de administración y su función es tomar decisiones, por eso recibe información mensual de los comités. Algunas decisiones requieren consulta de todos los socios. [Éstos] están informados a través de un boletín. Ahí se plantean problemas y alternativas. Cada zona toma su decisión. Si no hay consenso entonces decide el consejo de administración (entrevista con Gerardo Hernández, Grupo K'Nan Choch, abril 2002).

En la conversión del grupo a las actividades agroindustriales fortalece la relación entre el objetivo general de la organización — buscar alternativas orientadas a mejorar los ingresos de los socios - y el establecimiento del soporte organizacional adecuado para evaluar sus actividades, buscar información sobre tendencias del mercado y procesar esta información definiendo objetivos y movilizando sus recursos para cumplirlos. En ese momento, el funcionamiento interno de la organización (una de las expresiones de la dinámica institucional) permite establecer un campo de interacción propicio para compartir sus experiencias previas de comercialización. Además, este conocimiento tácito se reformula, median- 
te la exteriorización, en una expresión que orienta sus acciones futuras: agregar valor a las hortalizas. En ese momento es obvio que aún no existe diseño de un producto agroindustrial, ni siquiera una idea clara de cómo dirigirse hacia ese resultado, pero la expresión que formulan: agregar valor a las hortalizas, les permite orientar sus acciones posteriores y movilizar sus recursos.

En ese momento, el grupo inicia la construcción de vínculos de cooperación con diversas organizaciones con la finalidad de lograr su nuevo objetivo de agregar valor a su productos.

Para el diseño del producto se vinculan sucesivamente con la sede del Colegio Nacional de Educación Profesional (CONALEP) en el municipio de Tuxtla Chico y con el laboratorio químico privado Poliservicios Profesionales de Chiapas, ubicado en Tapachula (Poliservicios).

Con respecto a la experiencia del grupo con el CONALEP, ésta se limita a una propuesta de trabajo conjunto que nunca se concreta. En este sentido resulta sorprendente constatar que este plantel educativo cuenta con maquinaria para el procesamiento agroindustrial que se encuentra completamente subutilizada. Este equipo no se emplea para promover actividades de capacitación. Al no impulsarse estas actividades se desperdicia la oportunidad de fomentar procesos de aprendizaje en la práctica, los cuales constituyen espacios de interacción privilegiados para fomentar el desarrollo de la capacidad innovadora. Lo anterior revela, por otro lado, que la comunicación y coordinación entre organizaciones descansa sobre todo en iniciativas personales y no en marcos institucionales que las propicien. Esto no significa que se desvalorice el papel de dichas iniciativas, las cuales tienen un papel importante en la generación de las innovaciones; sin embargo, en la experiencia del grupo, la ausencia de marcos institucionales precisos propicia, en ocasiones, el surgimiento de prácticas abusivas en las relaciones con otras organizaciones.

Cuando el grupo inicia la relación con Poliservicios ya existe una idea general del tipo de producto que se requiere: hortalizas precocidas y congeladas. Esta definición se configura a partir de la demanda expresada por la empresa Superior Fud, la maquinaria de cocción a vapor que conocen los asesores en las instalaciones del CONALEP, además de folletos y manuales referidos a maquinaria de este tipo.

La relación con Poliservicios genera tres resultados: la demostración de que el producto puede venderse crudo y no congelado, el contacto con otra empresa para el diseño del empaque y la propuesta de actividades complementarias a la producción agroindustrial de hortalizas. En esta sección sólo se hará referencia al primer resultado.

El costo de los equipos de congelación hace inaccesible para el grupo desarrollar la producción de papas congeladas. Esta restricción financiera conduce a Poliservicios a explorar otras posibilidades de producción. La búsqueda es satisfactoria porque se demuestra que mediante un proceso de secado al alto vacío e inyección de nitrógeno es posible conservar las papas crudas. Estos resultados son la base para formular el proyecto 
de la planta industrial que será financiada fundamentalmente por conducto de la oficina regional del Fondo Nacional de Empresas en Solidaridad (FONAES), ubicada en Motozintla.

Debe señalarse que los resultados anteriores emergen de una relación poco estructurada entre el grupo y Poliservicios, rasgo que se manifiesta en la inexistencia de compromisos claros entre las partes y en la ausencia de sanciones si éstos no se cumplen.

La experiencia del grupo en esta etapa muestra la importancia que tiene la disponibilidad de información sobre el mercado de productos agroindustriales. Sin ella es imposible que las organizaciones identifiquen demandas que potencialmente pueden abastecer. Una vez que se identifican estos productos, también se requiere de servicios de asistencia técnica y financiera indispensables para el diseño inicial de los mismos. Esta etapa revela dificultades en la prestación de estos servicios. Esta situación se aprecia en la posición adoptada por CONACYT: negarse a asesorar el proyecto mientras no se estableciera una planta de producción. Cuando el grupo reestablece el contacto con el departamento de modernización tecnológica de esta dependencia, se condiciona la asesoría a los resultados de una evaluación tecnológica inicial (ETI). Es decir, la asesoría se otorga cuando el diseño del producto ya presenta un grado de desarrollo considerable.

A partir de los hechos que se registran en esta etapa podría formularse la siguiente proposición: en las regiones marginadas la dinámica institucional que se manifiesta, por ejemplo, en relaciones poco estructuradas entre las organizaciones o en desfases en la atención a sus necesidades, dificulta el establecimiento de vínculos entre actores, desincentiva la formación de redes y, con ello, desalienta el desarrollo de la capacidad innovadora.

La generalidad de la explicación la hace atractiva, pero al mismo tiempo revela su limitación, debido a su carácter estático. La forma en que la dinámica institucional incide en la formación y desarrollo de los ámbitos de interacción donde se intercambia conocimiento se modifica constantemente e imprime matices a la explicación general a la que se ha hecho referencia. Así puede apreciarse que en cierto momento la cohesión organizativa del grupo le permite formular con claridad el propósito de agregar valor a sus productos. Sin embargo, más tarde (como podrá apreciarse en el siguiente apartado) esta cohesión presenta fisuras y genera un comportamiento incongruente que repercutirá en el proceso de experimentación y rediseño. Del otro lado, a pesar de la laxitud de la relación con Poliservicios, no puede negarse que obtienen un diseño más preciso que se convierte en el punto de partida para el trabajo de experimentación y rediseño.

\section{Experimentación y rediseño}

Esta etapa tiene dos momentos fundamentales: el primero se refiere a la participación directa de CONACYT en el proyecto; el segundo comprende la fase final de experimentación y es responsabilidad exclusiva del equipo técnico del Grupo. Antes de abordar estos aspectos es necesario mencionar el marco en que se desarrolla esta etapa. 
Las primeras pruebas de producción, de resultados negativos, revelan problemas internos del grupo que se han ido acumulando y no han sido enfrentados adecuadamente. El problema de fondo es que el proyecto de industrialización no está plenamente asumido por la mayoría de los socios.

El proceso exitoso de socialización que desarrollan con anterioridad para evaluar sus experiencias de comercialización en fresco, no se lleva a cabo en este momento para intercambiar experiencias y percepciones sobre las implicaciones del proyecto de industrialización.

A la situación interna de la organización debe sumarse que las agencias internacionales como MISEREOR (organización para el desarrollo patrocinada por la conferencia episcopal de la iglesia católica alemana) y la IAF (Interamerican Foundation, organismo patrocinado por el senado de los Estados Unidos), que financian sus proyectos de producción de hortalizas orgánicas, se conforman con el cumplimiento de metas que garanticen el autoconsumo de los productores. Esta perspectiva desalienta cualquier preocupación por mejorar la calidad de las hortalizas. De nueva cuenta surgen evidencias del cambio permanente de los aspectos institucionales y su impacto en la generación de los ámbitos de interacción entre actores.

El principal resultado de la asesoría de CONACYT es la identificación de los problemas de corte y su efecto en la reducción de la vida del producto en anaquel. Este resultado es muy importante porque muestra a los asesores y directivos las implicaciones de no controlar la calidad de la papa que producen los socios. La asesoría de los consultores certificados por CONACYT empieza a mostrar deficiencias a partir del momento que se discute cómo corregir los problemas del corte. De acuerdo con los asesores, las observaciones de los consultores se tornan generales y poco fundamentadas y no permiten resolver los problemas identificados. Al respecto merece señalarse que no existe información sobre la supervisión de las actividades de estos consultores por parte del departamento de modernización tecnológica de CONACYT.

La asesoría de los consultores certificados por CONACYT termina en noviembre de 2000. A partir de ese momento, como señala uno de los asesores del grupo, se vuelven a quedar solos. El hecho relevante es que la experiencia que han acumulado les permite conducir con éxito la fase final de experimentación y rediseño del producto. Por un lado, elaboran un proyecto para comprar una máquina de corte adecuada. Una vez que disponen de este equipo, deciden contratar a una persona especializada para hacerse cargo de las pruebas finales, las cuales resultan satisfactorias en diciembre de 2001.

De esta última etapa, caracterizada por la experimentación y el rediseño del producto, destacan los siguientes aspectos.

En primer lugar, se aprecia la disposición permanente del grupo para vincularse con otras organizaciones y que han adoptado como lógica general de funcionamiento. Los 
alcances de esta lógica se aprecian, en segundo lugar, en los vínculos que establecen con CONACYT. Aun cuando esta institución presenta muchas objeciones para relacionarse con el grupo, finalmente coopera en la realización del proyecto "Implementación de los procesos de sanitización y vida de anaquel en la obtención de papas a la francesa para su consumo en fresco" y más adelante en el establecimiento del Centro de Desarrollo Tecnológico de Agricultura Orgánica K'Nan Choch, A.C. (Centro). Es decir, el grupo logra establecer un vínculo con el organismo nacional encargado de apoyar el desarrollo tecnológico. En tercer lugar, es muy importante señalar que la búsqueda de soluciones en el exterior para resolver los problemas de la producción agroindustrial, tiene como contraparte un descuido en la atención a los aspectos internos que inciden en la generación de esos problemas. Inclusive se aprecia que la búsqueda de asesoría externa se concentra en el ámbito de la producción agroindustrial y no en el del fortalecimiento institucional del grupo. En cuarto lugar, debe resaltarse que los asesores del grupo han desarrollado un largo proceso de aprendizaje en la práctica que, como ellos mismos señalan, les faculta para asesorar a otras organizaciones interesadas en producir bienes agroindustriales. De hecho, una de las funciones del Centro es la asesoría a otras organizaciones. Es decir, se crean condiciones organizativas formales que favorecen la difusión de la experiencia del grupo.

\section{Reflexiones finales}

Las discusiones contemporáneas sobre desarrollo regional revelan una concepción nueva del territorio. Éste no se concibe como depósito de recursos genéricos susceptibles de utilizarse, sino como ámbito de construcción de recursos específicos. De ahí que el potencial de desarrollo de una región no se considera dependiente de la identificación de sus recursos, sino como un proceso en función de la capacidad de sus actores para construir y movilizar el conjunto de sus recursos territoriales. Desde esta perspectiva, el estudio de la innovación no puede disociarse del proceso constructivo e interactivo anclado en un territorio, esto es, de la dinámica territorial.

En correspondencia con este punto de vista, enfoques como los del medio innovador y los sistemas de innovación revelan la diversidad de actores y tipos de conocimiento que intervienen en los procesos contemporáneos de innovación. Esta perspectiva permite abordar experiencias, como la analizada en este documento, cuya existencia ni siquiera podía ser reconocida mediante el uso de enfoques que restringen la actividad innovadora al ámbito de la I\&D. Además, el énfasis en el carácter interactivo de los procesos contemporáneos de innovación conduce a considerar aspectos que inciden en la formación y funcionamiento de las redes de cooperación que los hacen posible. Esto explica el interés en el papel de las instituciones, en su doble acepción de reglas de regulación social y organismos que la ejercen. 
El análisis de la experiencia del Grupo K'Nan Choch muestra el vínculo entre la generación de una innovación de producto y los mecanismos de cooperación que contribuyen a su desarrollo.

El marco que hace posible el análisis de dos etapas de la historia del grupo articula dos dimensiones que particularizan la noción de la dinámica territorial: la dinámica institucional y la interacción social entre los conocimientos tácito y explícito.

Se aprecia que este marco resulta útil para identificar los ámbitos específicos en los cuales los miembros del grupo y otras organizaciones intercambian su conocimiento y construyen, paulatinamente, una innovación de producto. Como señalan Maillat (1996) y Fischer (2001), la experiencia del grupo muestra que las innovaciones no resultan necesariamente de la materialización, sin fricciones, de una idea claramente concebida en un departamento de investigación que sólo necesita desarrollarse mediante el concurso de recursos humanos y tecnológicos disponibles en el mercado; por el contrario, las innovaciones se moldean en un proceso de interacciones que se desarrolla por tanteo, experimentándose aciertos y errores.

El análisis de la experiencia del grupo también muestra cómo la dinámica institucional incide en la formación y desarrollo de ámbitos donde se intercambia conocimiento. Esto se aprecia en los efectos diferenciados que ejercen los cambios en la cohesión del grupo durante el desarrollo del producto agroindustrial, así como en los resultados contrastantes de las relaciones con Poliservicios y CONACYT. Estos resultados muestran los problemas que genera la ausencia de acuerdos formales que sancionen incumplimientos.

El análisis de la experiencia del Grupo K'Nan Choch sugiere problemas que merecen mayor atención, entre ellos: valorar el efecto de las instituciones (formales y no formales) sobre los procesos de innovación requiere enfoques metodológicos que pongan de relieve su historicidad, es decir, que den cuenta de su proceso de formación y de los cambios que registren, en lugar de suponer que son normas establecidas y rígidas. Al respecto resultan sugerentes las discusiones expuestas por la economía institucional y evolutiva (Hodgson, 1999 y 2002).

\section{Bibliografía}

Asheim, Bjorn T. y Philip Cooke, "Local Learning and Interactive Innovation Networks in a Global Economy”, en E. J. Malecki y P. Oinas (editores) Making Connections. Technological learning and regional economic change, Aldershot, Ashgate, 1998, pp. 145-178.

Becattini, Giacomo y Enzo Rullani, "Local systems and global connections: The role of knowledge", en F. Cossentino et al. (editores) Local and regional response to global pressure: The case of Italy and its industrial districts, Génova, International Institute of Labour Studies, 1996, pp. 159-174.

Beckmann, Martin J. y Jacques-François Thisse, "The Location of Production Activities", en P. Nijkamp (ed.) Handbook of Regional and Urban 
Economics, vol. 1, Regional Economics, Amsterdam, Holanda, 1986, pp. 21-95.

Bramanti, Alberto y Mario Maggioni (editores), La dinamica dei sistemi produttivi territoriali: teorie, tecniche, politiche, Milán, Franco Angeli, 1997.

Bramanti,Alberto y Remigio Ratti, "The Multi-Faced Dimensions of Local Development", en A. Bramanti et al. (editores) The Dynamics of Innovative Regions. The GREMI Approach, Aldershot, Ashgate, 1997.

Conti, Sergio y Michael Taylor (editores), Interdependent and Uneven Development. Globallocal perspectives, Aldershot, Ashgate, 1997.

Cossentino, Francesco et al. (editores), Local and regional response to global pressure: The case of Italy and its industrial districts, Génova, International Institute of Labour Studies, 1996.

Cooke, Philip y Kevin Morgan, The Associational Economy. Firms, Regions and Innovation, Oxford, Oxford University Press, 2000.

Edquist, Charles (editor), Systems of Innovation. Technologies, Institutions and Organizations, Londres, Pinter, 1997.

Echeverri-Carroll, Elsie L. y William Brennan, "Are Innovation Networks Bounded by Proximity?", en M. M. Fischer et al. (editores) Innovation, Networks and Localities, Berlín, SpringerVerlag, 1999, pp. 28-49.

Fischer, Manfred M. y Josef Frölich (editores), Knowledge, Complexity and Innovations Systems, Berlín, Springer-Verlag, 2001.

Fischer, Manfred M., "The Innovation Process and Network Activities of Manufacturing Firms", en M. M. Fischer et al. (eds.) Innovation, Networks and Localities, Berlín, SpringerVerlag, 1999, pp. 11-27.

_- "Innovation, knowledge creation and systems of innovation" The Annals of Regional Science, 35 (2), 2001, pp.199-216.

Hodgson, Geoffrey M., Evolution and Institutions. On Evolutionary Economics and the Evolution of Economics, Cheltenham, Edward Elgar, 1999.

_- (editor), A Modern Reader in Institutional and Evolutionary Economics. Key Concepts, Cheltenham, Edward Elgar, 2002.

Maillat, Denis, Du district industriel au milieu innovateur: contribution a une analyse des organisations productives territorialisées, Neuchâtel, IRER, 1996, http://www.unine.ch/irer/ wp9606a.doc.

_ y Laila Kebir, Learning region et systèmes territoriaux de production, Neuchâtel, IRER, 1998, http://www.unine.ch/irer/wp9802b. doc.

- y Nicolas Grosjean, "Globalisation and Territorial Production Systems", en M. M. Fischer et al. (editores) Innovation, Networks and Localities, Berlín, Springer-Verlag, 1999, pp. 50-65.

Malecki, Edward J. y Päivi Oinas (editores), Making Connections. Technological learning and regional economic change, Aldershot, Ashgate, 1998.

Metcalfe, Stan, J., "The Design of Order. Notes on Evolutionary Principles and the Dynamics of Innovation", Revue Économique, vol. 46, núm. 6, noviembre, 1995, pp. 1561-1583.

Morales, Federico, "Procesos de innovación en la Sierra de Chiapas: redes e instituciones", reporte final de investigación del proyecto Dinámica estructural en economías regionales subdesarrolladas: un marco de interpretación de la relación global-local, San Cristóbal de Las Casas, PROIMMSE-IIA-UNAM, 2003, pp. 160-237.

Moulaert, Frank y Farid Sekia, "Innovation region, social region? An alternative view of regional innovation", European Meeting on Applied Evolutionary Economics, Grenoble, 7-9 junio, mimeo, 1999.

Nonaka, Ikujiro y Hirotaka Takeuchi, La organización creadora del conocimiento. Cómo las compañías japonesas crean la dinámica de la innovación, México, Oxford University Press, 1999.

Observatorio Europeo LEADER, La competitividad de los territorios rurales a escala global. Cuaderno de la Innovación núm. 6, Fascículo 5, febrero, 2001.

Oman, Charles, Globalisation and Regionalisation: The Challenge for Developing Countries, Paris, OECD, 1994.

Rullani, Enzo, "Piú locale e piú globale: verso una economia postfordista del territorio", en Bramanti, A. y M. Maggioni, (editores) La dinamica dei sistemi produttivi territoriali: teorie, tecniche, politiche, Milán, Franco Angeli, 1997, pp. 85-111.

Takayama, T. y Walter C. Labys, "Spatial Equilibrium Analysis" en P. Nijkamp, (editor) Handbook of Regional and Urban Economics, vol. I: Regional Economics, Amsterdam, Holanda, 1986, pp.171199.

Vázquez, Antonio, Política económica local, Madrid, Pirámide, 1993.

, Desarrollo, redes e innovación. Lecciones sobre desarrollo endógeno, Madrid, Pirámide, 1999.

Veltz, Pierre, "D'une géographie des coûts à une géographie de l'organisation. Quelques thèses sur l'évolution des rapports entreprises/ territoires", Revue Économique, vol. 44, núm. 4, julio, 1993, pp. 671-684.

Vence, Xavier, Economía de la innovación y del cambio tecnológico, México, Siglo xxi, 1995. 